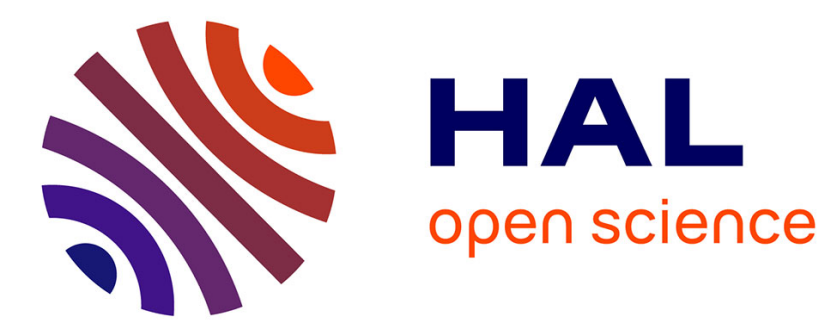

\title{
Contribution to the nonlinear theory of plasma wave excitation inside the electron foreshock region
}

\author{
V. I. Sotnikov, V. Fiala, Pierrette Décréau, V. V. Pivovarov, I. V Shapiro
}

\section{To cite this version:}

V. I. Sotnikov, V. Fiala, Pierrette Décréau, V. V. Pivovarov, I. V Shapiro. Contribution to the nonlinear theory of plasma wave excitation inside the electron foreshock region. Journal of Geophysical Research Space Physics, 1994, 99 (A12), pp.473 - 496. 10.1029/94JA01403 . insu-02873958

\section{HAL Id: insu-02873958 \\ https://hal-insu.archives-ouvertes.fr/insu-02873958}

Submitted on 19 Jun 2020

HAL is a multi-disciplinary open access archive for the deposit and dissemination of scientific research documents, whether they are published or not. The documents may come from teaching and research institutions in France or abroad, or from public or private research centers.
L'archive ouverte pluridisciplinaire HAL, est destinée au dépôt et à la diffusion de documents scientifiques de niveau recherche, publiés ou non, émanant des établissements d'enseignement et de recherche français ou étrangers, des laboratoires publics ou privés. 


\title{
Contribution to the nonlinear theory of plasma wave excitation inside the electron foreshock region
}

\author{
V. I. Sotnikov, ${ }^{1}$ V. Fiala, ${ }^{2}$ and P. M. E. Décréau \\ Laboratoire de Physique et Chimie de l'Environnement terrestre et planétaire, CNRS, Orléans \\ France
}

V. V. Pivovarov
Space Research Institute, Moscow, Russia

I. V. Shapiro

International Institute for Mathematical Geophysics, Moscow, Russia

\begin{abstract}
The broadband electrostatic waves inside the electron foreshock region well away from its boundaries were explained as a beam mode for which the beam velocity is lower than the thermal velocity of the background plasma. We discuss here the nonlinear saturation of this instability. The waves propagating along the beam are stabilized by a plateau formation on the electron distribution function. The waves that propagate at an angle to the beam are stabilized in a different way. In the course of the development of the instability the beam velocity spreads and an initially negative energy mode, which is initially unstable, experiences increased damping by the beam particles and finally is stabilized due to this effect. We give an estimation of the wave energy in both cases, and we present a numerical modelling to substantiate our analytical estimates. The saturated wave energy level is found to be comparable in both cases and agrees with the levels observed in the foreshock.
\end{abstract}

\section{Introduction}

Two kinds of waves have been observed in the electron foreshock [Etcheto and Faucheux, 1984]. Waves at the plasma frequency excited by the bump-on-tail distributions are observed close to the foreshock upstream edge [Fitzenreiter et al., 1984, 1990] and a broadband electrostatic noise below the local plasma frequency is observed deep inside the foreshock, far away from its boundaries [Lacombe et al., 1985; Onsager and Holzworth, 1990]. This broadband noise is well explained by the beam-driven electron sound oscillations [ Fuselier et al., 1985, Marsh, 1985, Gary, 1985](see also Burinskaya and Meister [1989], Shriver and Ashour-Abdalla [1989], and Dum [1990]). A beamlike distribution in the foreshock arises due to the reflection and acceleration of solar wind electrons at the Earth's bow shock. A kinematic argument of Filbert and Kellog [1979] (see also

\footnotetext{
${ }^{1}$ Now at Institute of Geophysics and Planetary Physics, University of California, Los Angeles.

${ }^{2}$ Now at Institute of Atmospheric Physics, Czech Academy of Physics, Prague, Czech Republic.
}

Copyright 1994 by the American Geophysical Union.

Paper number 94JA01403. 0148-0227/94/94JA-01403\$05.00
Cairns [1987]) shows that the reflected electrons must have a velocity greater than

$$
v_{\mathbf{b}}=v_{n} \frac{R}{X}
$$

well inside the foreshock. Here $v_{n}$ is the solar wind velocity component perpendicular to the magnetic field, $R$ is the distance from the bow shock measured along the magnetic field, $X$ is in the direction perpendicular to it, measured from the foreshock's upstream edge. This gives rise to a beam like electron distribution with the velocity $v_{b}$. Inside the foreshock this beam velocity is lower than the thermal velocity $v_{T e}$ of the background solar wind plasma electrons and the beam density $n_{b}$ is weak, $n_{b} \ll n_{o}$, where $n_{o}$ is the background solar wind plasma density.

The aim of this paper is to discuss the nonlinear development of the beam-driven electron acoustic instability. Namely, we will show that the waves are stabilized by two essentially different processes. The waves going along the beam saturate as the plateau develops on the electron distribution function. The waves that propagate at an angle to the beam are stabilized as the beam velocity spreads and grows as the beam is being thermalized. This leads to enhanced damping by the beam electrons and the wave growth is switched off. We investigate the model problem and examine separately the resonant quasi-linear diffusion of the Maxwellian 
background plasma and the nonlinear interaction of the beam electrons with the excited wave spectrum. Next we define the most important nonlinear effect responsible for the stabilization of the instability using the parameters typical for the electron foreshock region. Both components, that is, background solar wind electrons and beam electrons are in resonance with the same waves, but every solar wind electron is in resonance only with several waves from the unstable phase velocity interval. At the same time, every beam electron, due to the condition $\Delta v_{b} \ll \gamma / k$, is in resonance with the whole unstable wave spectrum. So it is because the nonlinear interaction of the excited wave spectrum with these two components takes place in different ways that we decided to separate the two distributions. We investigate the nonlinear interaction of waves with each of these components, but the nonlinear influence of one component on the other through the waves is neglected in the paper.

In the following section we consider the one dimensional diffusion of plasma electrons in velocity space. The quasi-linear set of equations that describes the process is solved and the resulting wave spectrum is calculated. In section 3 the nonlinear dynamics of oblique waves is analyzed. In this case the formation of the plateau cannot stop the instability since particles with velocities much larger than the thermal velocity $v_{T e}$ are in resonance with the oblique waves. The condition for quenching the instability is found by looking how the growing spread in transverse velocities of the beam electrons lead to the reduction of the growth rate. A method of incomplete numerical simulation [Matsiborko et al., 1972] is used in section 4 to substantiate our analytical findings. In this procedure only the beam particles (the spreading of their velocities) is taken into account, the distribution of the plasma electrons is held constant. Both the cases of one dimension and two dimensions are treated. Then we discuss and compare the analytical and numerical results. Our findings are in a satisfactory agreement with the observations in what concerns the frequency spectrum and typical wave amplitudes.

\section{Electron Acoustic Beam-Driven Instability in the Solar Wind}

We shall consider electrostatic oscillations of the solar wind electrons in the case when the electron distribution function has a $\delta$-type singularity at a velocity $v_{b} \ll$ $v_{T e}$. In this case the distribution function represented qualitatively in Fig. 1 can be written in the following form:

$$
f_{e}(\vec{v})=f_{o}(\vec{v})+n_{b} \cdot \delta\left(\vec{v}-\vec{v}_{b}\right), \quad v_{b} \ll v_{T e},
$$

where $n_{b}$ and $v_{b}$ are the density and the speed of beam electrons, respectively, $v_{T e}=\left(K T_{e} / m\right)^{1 / 2}$ is the thermal velocity of background plasma electrons, $K$ is Boltzman's constant, and $f_{o}(\vec{v})$ is the distribution function of the solar wind electrons, assumed to be Maxwellian.

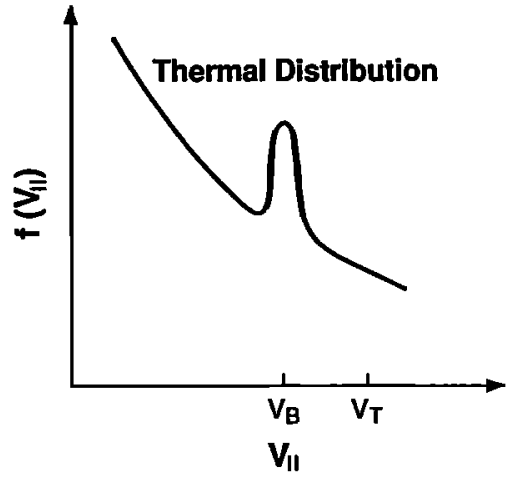

Figure 1. Qualitative electron distribution function considered.

The electrostatic oscillations under consideration have the following dispersion relation [Mikhailovskii, 1974]:

$$
1+\frac{1}{k^{2} r_{D e}^{2}}\left(1+i \sqrt{\frac{\pi}{2}} \frac{\omega}{k v_{T e}}\right)-\frac{\omega_{b}^{2}}{\left(\omega-k_{\|} v_{b}\right)^{2}}=0,
$$

where $r_{D e}$ is the background electron Debye radius and $k, k_{\|}$are the absolute values of the wave vector and of its component parallel to the direction of the beam propagation, $\omega_{b}=\left(n_{b} e^{2} / \epsilon_{0} m\right)^{1 / 2}$ is the plasma pulsation of the beam.

The solution of the dispersion relation gives both the pulsation and the growth rate of unstable oscillations. Since the imaginary part of $\omega$ is small compared to its real part $\omega_{r}$, the notation $\omega$ is used in place of $\omega_{r}$.

$$
\begin{gathered}
\omega=k_{\|} v_{b}-\omega_{b} \frac{k r_{D e}}{\left(1+k^{2} r_{D e}^{2}\right)^{1 / 2}} \\
\gamma=\sqrt{\frac{\pi}{8}} \omega \frac{\omega_{b}}{\omega_{p}\left(1+k^{2} r_{D e}^{2}\right)^{3 / 2}},
\end{gathered}
$$

where $\omega_{p}$ is the plasma pulsation in the background solar wind electrons. The threshold for the onset of instability,

$$
v_{b} \geq \sqrt{\frac{n_{b}}{n_{o}}} \frac{k}{k_{\|}} v_{T e}
$$

defines quite a wide interval for angles of propagation of unstable waves from parallel to almost normal propagation. A possible nonlinear mechanism that can stabilize the wave growth is the velocity diffusion of plasma electrons in resonance with the unstable waves. In this case every unstable wave on the distribution function of the background plasma singles out it's own group of the resonance particles. The resonance interaction between the waves and beam particles takes place in another way. Because of the condition $\Delta v_{b} \ll \gamma / k$ all the beam as a whole is in resonance with every harmonic of the unstable wave packet. The velocity diffusion leads to the formation of a plateau in the resonant part of the velocity distribution function of the background electrons due to which the instability of the negative energy mode is quenched. The quasi-linear set of equations describing this process has the following form: 


$$
\begin{aligned}
\frac{\partial f}{\partial t} & =\frac{e^{2}}{m^{2}} \frac{\partial}{\partial v}\left[\frac{\left|E_{k}\right|^{2}}{\left|v-\frac{d \omega}{d k}\right|} \frac{\partial f_{o}}{\partial v}\right] \\
\frac{\partial\left|E_{k}\right|^{2}}{\partial t} & =\frac{\omega_{b} v_{T e}^{2}}{4 \epsilon_{0} n_{o}} \frac{k r_{D e}}{\left(1+k^{2} r_{D e}^{2}\right)^{3 / 2}} \frac{\partial f_{o}}{\partial v}
\end{aligned}
$$

where $f$ is the slowly varying distribution function of the quasi-linear theory. The relation between the resonance velocity $v$ and the wavenumber $k$ is determined by the dispersion law:

$$
v=v_{b}-\frac{\omega_{b} r_{D e}}{\left(1+k^{2} r_{D e}^{2}\right)^{1 / 2}}
$$

and the group velocity of the unstable waves is

$$
\frac{d \omega}{d k}=v_{b}-\frac{\omega_{b} r_{D e}}{\left(1+k^{2} r_{D e}^{2}\right)^{3 / 2}}
$$

The quasi-linear equations (4) and (5) are written for the one-dimensional case where only waves propagating in the beam direction are excited and the formation of the plateau in the resonant part of the distribution function is the dominant nonlinear process. The discussion of the role of a plateau formation for the twodimensional case with the excitation of oblique waves is carried out below in section 3 .

We can investigate the plateau formation using the standard technique (see, for example, Akhiezer et. al. [1975]). In our case the distribution function is discontinuous on the ends of the unstable phase velocity interval. It is possible in principal to avoid this discontinuity by using an effective collision frequency instead of the binary collisions. For the instability under question the quasi-linear evolution of the distribution function takes place in two directions from the point $v_{0}=v_{b}-v_{T e}\left(2 n_{b} / 3 n_{0}\right)^{1 / 2}$. This point corresponds to the maximum of the growth rate. Dynamics of the plateau formation for the similar physical situation of the ion-beam driven acoustic instability was investigated by Burinskaya and Meister [1989]. In our case of the electron beam-driven acoustic instability this corresponds to the unstable phase velocity range $\left(v_{\min }=v_{b}-\omega_{b} r_{D e}, v_{\max }=v_{b}\right)$. In the paper by $D u m$ [1990] the process of the plateau formation was investigated with the help of numerical simulations. It was shown that plateau formation takes place in the region of phase velocities slightly larger than $v_{b}$. We, from the very beginning, examine the instability using an extremely narrow distribution of the beam electrons in velocity space ( $\delta$ function distribution in velocity space for the beam particles). In this case the unstable phase velocity interval is restricted by $v_{b}$ in it's upper limit. We then find the plateau formation on the resonant bulk solar wind distribution by neglecting velocities $>v_{b}$. These velocities can appear due to the beam velocity spread and were investigated separately so that there would be no contradiction with the particle conservation law. We also neglect in our model the nonresonant diffusion of bulk solar wind electrons since it cannot play a significant role in the electron foreshock region where the collision frequency is very small.
The energy integral of the quasi-linear set of equations (4) and (5) has the form:

$$
\begin{array}{r}
\left|E_{k}\right|^{2}=\frac{\pi K T_{e}}{\epsilon_{0}} \frac{\omega_{b}}{\omega_{p}^{2}} \frac{k r_{D e}}{\left(1+k^{2} r_{D e}^{2}\right)^{3 / 2}}|v-d \omega / d k| \\
\cdot \int_{v_{\min }}^{v}\left(f-f_{o}\right) d v
\end{array}
$$

where $K T_{e}$ is the electrons' thermal energy and $v_{\min }$ the minimal value of the resonant velocity:

$$
v_{\min }=v_{b}-\omega_{b} r_{D e} \quad(k \rightarrow 0)
$$

In the one-dimensional case, a plateau is formed in the velocity interval $v_{\min }<<v<v_{b}$. The height of the plateau can be found from the number of particles conservation law:

$$
f^{\infty}=\frac{1}{v_{b}-v_{\min }} \int_{v_{\min }}^{v_{b}} f_{o} d v
$$

where $f_{o}$ is the Maxwellian velocity distribution, which can be written in the following form for the velocities $v \ll v_{T e}$ :

$$
f_{o}=\frac{n_{o}}{\sqrt{2 \pi} v_{T e}} \cdot\left(1-v^{2} / 2 v_{T e}^{2}\right)
$$

Then,

$$
f^{\infty}=\frac{n_{o}}{\sqrt{2 \pi} v_{T e}}\left[1-\frac{v_{b}^{2}+v_{b} v_{\min }+v_{\min }^{2}}{6 v_{T e}^{2}}\right]
$$

and from (7) the following formula for the wave spectral density at the final stage of plateau formation can be obtained

$$
\begin{array}{r}
\left|E_{k}\right|^{2}=\frac{\pi n_{o} K T_{e} r_{D e}}{\epsilon_{0}} \frac{\omega_{b}^{4}}{\omega_{p}^{4}}\left(\frac{m v_{b}^{2}}{8 \pi K T_{e}}\right)^{\frac{1}{2}} \frac{k^{3} r_{D e}^{3}}{\left(1+k^{2} r_{D e}^{2}\right)^{\frac{7}{2}}} \\
\cdot\left[1-\frac{1}{\sqrt{1+k^{2} r_{D e}^{2}}}\right]
\end{array}
$$

An integration over $k$ gives the following wave energy level for the beam-driven electron sound mode:

$$
W_{E}=\sum_{k} \epsilon_{0}\left|E_{k}\right|^{2} \simeq \frac{n_{o} K T_{e}}{40 \sqrt{2 \pi}} \frac{n_{b}^{2}}{n_{o}^{2}} \frac{v_{b}}{v_{T e}}
$$

It should be noted that there exists another mechanism for the saturation of the wave growth. The instability under consideration is the negative energy mode instability which exists only in the case of the monoenergetic beam. The growth of the beam velocity spread can stabilize the instability. This happens when damping produced by the beam electrons due to the velocity spread will exceed the growth rate connected with the resonant interaction with the background electrons. To find this critical value of the beam velocity spread, we can include the damping due to the beam particles into the dispersion equation and from the numerical solution try to find the maximum possible beam velocity spread. We can introduce the parameter $\delta=\Delta v_{b \max } / v_{T e}$ where $\Delta v_{b \max }$ is the maximum value of the beam velocity spread. From the requirement that the growth rate has 
to be positive it is possible to get the scaling [Dum, 1989]:

$$
\delta \sim \eta \sqrt{n_{b} / n_{e}}
$$

where the parameter $\eta \ll 1$ depends on the ratio $\omega / k v_{T e}$. For the ratio of the beam velocity to the background electron speed, we use $v_{b} / v_{T e} \sim 0.5$, which is typical inside the electron foreshock region. This gives us $\eta \sim 0.2$. As it is well known, the growth of thermal energy in the beam caused by waves is equal to the kinetic energy of the beam particles oscillating in the wave field:

$$
\frac{m\left(\Delta v_{b}\right)^{2}}{2}=\frac{e^{2}}{2 m} \sum_{k} \frac{\left|E_{k}\right|^{2}}{\delta \omega_{k}^{2}}
$$

where $\delta \omega_{k}=\omega-k_{z} v_{b}$. With the use of (2) and condition (10) we can obtain an estimate for the energy of the wave electric field that is sufficient for the saturation of the instability as the result of the growth of the beam velocity spread. This is achieved by restricting the summation over $k$ to the values which correspond to the maximum of the growth rate, that is for $k r_{D e} \simeq 1$ :

$$
W_{E} \simeq \sum_{k} \epsilon_{0}\left|E_{k}\right|^{2} \simeq \eta^{2} n_{0} K T_{e} \frac{n_{b}^{2}}{n_{o}^{2}}
$$

The estimate (12) was obtained in the paper by Dum [1989]. When this estimate is compared to (9) it follows that in the one-dimensional case the velocity diffusion of plasma electrons and the plateau formation is the dominant nonlinear process when compared to the negative energy mode instability. It stabilizes the instability for amplitudes that are somewhat smaller (by a factor $v_{b} /\left[40(2 \pi)^{1 / 2} v_{T e} \eta^{2}\right]$ ) for the quasi-linear wave energy. Nevertheless, the growth of the velocity spread is significant in the final stage. This is confirmed by a one-dimensional numerical simulation of the instability by Dum [1990]. In this paper the author does not separate the distributions of the background solar wind plasma and beam electrons. This makes it difficult to make the comparison with some of our results where this separation was used. One of the main differences for the runs with $v_{b} / v_{T e}=1.25$, which is the closest to our case of $v_{b} / v_{T e} \simeq 0.5$, is connected with the appearance of the particle diffusion into the region $v>v_{b}$. This can be explained, if we take into account the velocity spread of the beam electrons.

It is also necessary to point out that the dependence of the kinetic instability of the downshifted oscillations from the $v_{b} / v_{T e}$ ratio was examined in the paper by Dum [1989]. Also, it should be noted that in the twodimensional simulation the plateau formation cannot stabilize the instability, and in this case the saturation is only due to the growth of the velocity spread in the beam. This case is the subject of the next section.

\section{Nonlinear Saturation of the Instability in the Two-Dimensional Case}

The dispersion equation for waves that propagate at an angle to the beam has still the form (2) and the nec- essary condition for the instability $(\omega>0)$ determines possible angles of propagation of unstable waves derived from equation (3):

$$
\frac{k_{\|}}{k} \geq \frac{\omega_{b}}{\omega_{p}} \frac{v_{T e}}{v_{b}}
$$

Also, in the two-dimensional case the phase velocity may attain very low values and the width of the interval of the resonant particle phase velocities $\Delta v$ widens and becomes comparable to the beam velocity, $\Delta v \simeq v_{b}$. Under experimental conditions with dilute beam plasma $\left(\omega_{b} \ll \omega_{p}\right)$, the right hand side of the inequality (13) can be of the order of $1 / 10$. In which case, the waves propagating almost transverse to the beam are excited. For such waves the stabilization due to a plateau formation is impossible as it would be necessary to form a plateau in an infinite velocity interval $v_{\perp} \geq \omega / k$ over which the integration in the formula for the growth rate is carried out [Galeev and Sagdeev, 1983].

The instability in the two-dimensional case is stabilized by the growth of the transverse velocity spread in the beam. Again, as in the one-dimensional case, from the condition for the growth rate to be positive it is possible to get the scaling (10), but now the parameter $\eta$ will be smaller ( $\eta \leq 0.1$ ) because it depends on the ratio $\omega / k_{v e}$ and for the oblique waves this ratio is smaller. The growth of the transverse energy in the beam, equal to the kinetic energy of the beam particles oscillating in the wave electric field, gives

$$
\frac{m\left(\Delta v_{\perp}\right)^{2}}{2} \simeq \frac{e^{2}}{2 m} \sum_{k}\left|E_{k}\right|^{2} \frac{1}{\delta \omega_{k}^{2}}
$$

Now, using (14) one obtains the following result for the level of the wave energy at which the instability of oblique waves is stabilized:

$$
W_{E}=\sum_{k} \epsilon_{0}\left|E_{k}\right|^{2} \simeq \eta^{2} K T_{e} \frac{n_{b}^{2}}{n_{o}}
$$

As we shall see from the results of numerical simulations, the parameter $\eta^{2}$ is equal to $\eta^{2} \sim 10^{-2}$ (see next section).

\section{Incomplete Numerical Simulations in One- and Two-Dimensional Cases}

The process of saturation of the instability due to the increase of the transverse beam velocity spread can be described with the use of the method of incomplete numerical simulations [Matsiborko et al., 1972]. According to this method, we consider the distribution function of plasma electrons to be constant, and we follow the motion of the beam electrons during the development of the instability. We choose the initial distribution function of the beam electrons as

$$
f_{o b}=\frac{n_{b}}{2 \pi} \delta\left(v_{\perp}^{2}\right) \delta\left(v_{z o}-v_{b}\right)
$$

where $v_{z o}$ is the velocity component along the beam. The electric potential of the unstable wave packet cor- 
responding to a number of harmonics $k$ is represented in the following form:

$$
\Phi=\frac{1}{2} \sum_{\vec{k}}\left(\Phi_{k} e^{i \vec{k} \vec{r}-i \omega_{k} t}+\Phi_{k} e^{-i \vec{k} \vec{r}+i \omega_{k} t}\right)
$$

It obeys the Poisson equation:

$$
\varepsilon_{e} k^{2} \Phi_{k}=-4 \pi e \int f_{b} d^{3} v
$$

where $f_{b}$ is the current stage of the velocity distribution function in the beam, with the dielectric function:

$$
\varepsilon_{e}=1+\frac{1}{k^{2} r_{D e}^{2}}\left[1+i \sqrt{\frac{\pi}{2}} \frac{\omega_{k}}{k v_{T e}} \exp \left(-\omega_{k}^{2} / 2 k^{2} v_{T e}^{2}\right)\right]
$$

and with the source term $-4 \pi e \int f_{b} d^{3} v$ due to the beam electrons. The potential harmonics and the equations of motion for the beam electrons are given by

$$
\begin{gathered}
\Phi_{k}=-\frac{8 \pi e}{k^{2} \varepsilon_{e}} \frac{1}{N_{p}} \sum_{p} n_{p}\left(\vec{r}_{o p}\right) e^{-i \vec{k} \vec{r}_{p}+i \omega_{k} t} \\
\frac{d^{2} \vec{r}_{p}}{d t^{2}}=-\frac{e}{m} \sum_{\vec{k}} \vec{k} I_{m}\left(\Phi_{k} e^{i \vec{k} \vec{r}_{p}-i \omega_{k} t}\right)
\end{gathered}
$$

$N_{p}$ is the total number of the beam particles over a wave length and periodic boundary conditions are applied. The value $n_{p}\left(\vec{r}_{o p}\right)$ is the density of beam particles at initial positions $\vec{r}_{o p}$ and $\vec{r}_{p}(t)$ the position at time $t$ of an individual beam electron $p$. This set of equations was solved numerically in order to obtain the nonlinear saturation of the instability due to the increase of the beam velocity spread with the simulation system size equal to the maximum unstable wavelength. A nonuniform initial density of the beam electrons insures the presence of non linear terms in right-hand side of (20) for the initial steps of integration (after several steps, the density becomes self-consistantly inhomogeneous, due to the interaction with the excited electric field).

The results of simulations for the one-dimensional case (i.e., only the waves propagating along the beam

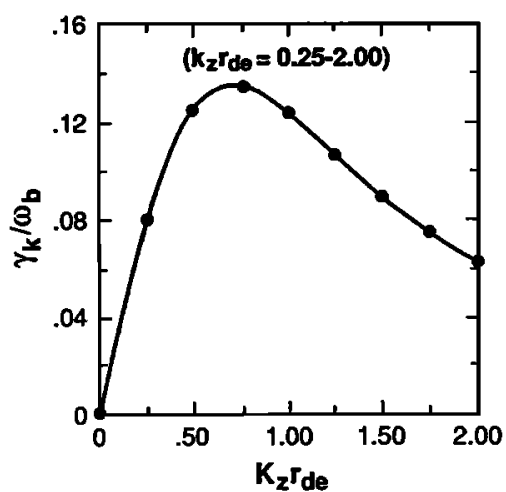

Figure 2. Parallel propagation: normalized growth rate versus normalized wavenumber, for $n_{b} / n_{o}=0.001$, $v_{b} / v_{T e}=0.5$.

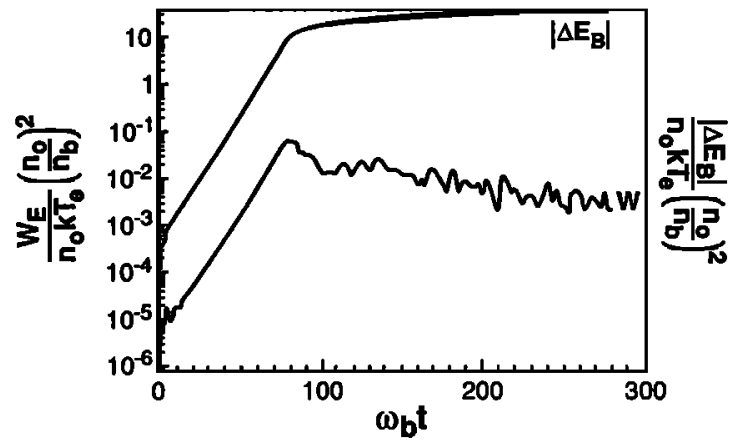

Figure 3. Parallel propagation: normalized to $n_{0} K T_{e}\left(n_{b} / n_{0}\right)^{2}$, the wave energy density $W_{E}$ and the change of the absolute value of beam kinetic energy $\left|\Delta E_{B}\right|=m\left(v_{b}^{2} / 2-<v^{2} / 2>\right)$, versus time. The chosen beam and plasma characteristics are as in Figure 2.

are considered) are presented in Figures 2 and 3. The growth rate versus the parallel wave number is shown in Figure 2, and the harmonics used in the simulation are marked. Figure 3 shows the growth of energy $W$ in the excited wave spectrum with time and the change of the beam particle kinetic energy related to the velocity spreading. From this figure we conclude that the wave energy saturates at

$$
W \sim 0.05\left(\frac{n_{b}^{2}}{n_{o}^{2}}\right) n_{o} K T_{e}
$$

due to the velocity spreading in the beam. As this value is higher than the one given by (9) for the saturation of instability by a plateau formation, it may be concluded that it is the plateau formation that stabilizes the waves going along the beam. This estimate appears to be in good agreement with the estimation (12) of the saturation by velocity spreading.

In the two-dimensional simulation case, Figure 4 shows the lines of constant level for the growth rate in the $k_{\|}$ (or $k_{x}$ ) and $k_{\perp}$ (or $k_{x}$ ) plane and the harmonics used. Taking into account the fact that due to the condition

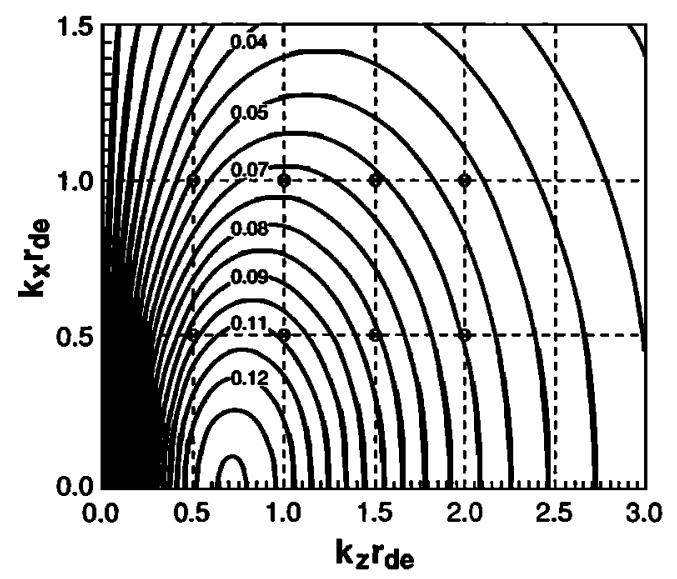

Figure 4. Oblique propagation: constant levels of normalized growth rate in the $k_{z}$ (parallel wave number) and $k_{x}$ (perpendicular wave number) plane. Same characteristics as in Figure 2. 


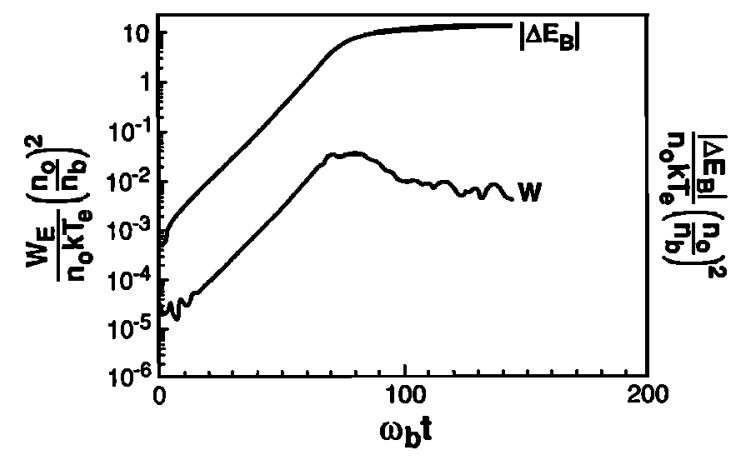

Figure 5. Same as Figure 3, for oblique propagation

$\Delta v_{b} \ll \gamma / k$ every beam particle is in resonance with the excited wave spectrum, we can conclude that the spacing of resonance velocities is smaller then the resonance width for the examined modes. Figure 5 is similar to Figure 3 , in the case of oblique waves. We conclude once again that our estimate (15) of the saturation level is in good agreement with the numerical results.

When we compare (15) and (9), we can see that both the waves going along and across the beam are stabilized at approximately the same level, even if the mechanisms involved in the saturation are different.

\section{Conclusions}

In the course of this work we have shown that the nonlinear saturation process for the beam driven electron acoustic instability for the waves propagating along the beam differs from that for oblique waves. Nevertheless, we have found that the saturated wave level is approximately the same in both cases. Now, if we take typical electron foreshock parameters in our formulas (9) and (15), we obtain a ratio between the wave electric field energy and the plasma energy $W / n_{0} K T_{e} \simeq 10^{-6}$ to $10^{-8}$, which is in satisfactory agreement with experimental data (see, for example, Etcheto and Faucheux [1984], Fitzenreiter et al. [1984, 1990], and Buckley et al. [1993]). Our results confirm also the conclusion made by Dum [1990], namely that for the waves along the beam, the main nonlinear effect is the quasi-linear transformation of the plasma electron distribution function. For the waves propagating across the beam it is the transformation of the beam electron distribution and increased damping by the beam particles which stabilizes the instability.

Acknowledgments. The authors acknowledge helpful discussions with M. Ashour-Abdalla.

The Editor thanks C.T. Dum and David Schriver for their assistance in evaluating this paper.

\section{References}

Akhiezer, A.I., I.A. Akhiezer, R.V. Polovin, A.G. Sitenko, and K.N. Stepanov, Plasma Electrodynamics, vol.2, p.54, Pergamon, New york, 1975.
Buckley, A.M., P.J. Christiansen, S.C. Chapman, and P. Devine, Beam-plasma simulations of varying wave dispersion applied to the Earth's electron foreshock,Eos Trans. $A G U, \gamma / 4,494,1993$.

Burinskaya, T.M., and C.V. Meister, Contribution for the ion-beam acoustic instability to the generation of broadband electrostatic noise in the Earth's magnetotail, Planet. Space Sci., 37, 145, 1989.

Cairns, I.H., The electron distribution upstream from the Earth's bow shock, J. Geophys. Res., 92, 2315, 1987.

Cairns, I.H., and S.F. Fung, Growth of electron plasma waves above and below $f_{p}$ in the electron foreshock, J. Geophys. Res., 93, 7307,1988.

Canu, P., Linear study of the beam-plasma interaction as a source mechanism for the broadband electrostatic emissions observed in the electron foreshock, J. Geophys. Res., 94, 87, 1989.

Dum, C.T. , Transition in the dispersive properties of beamplasma and two-stream instabilities, J. Geophys. Res., 94, 2429, 1989.

Dum, C.T., Simulation studies of plasma waves in the electron foreshock: The generation of downshifted oscillations, J. Geophys. Res., 95, 8123, 1990.

Etcheto, J. and M. Faucheux, Detailed study of electron plasma waves upstream of the Earth's bow shock, J. Geophys. Res., 89, 6631, 1984.

Filbert, P.C., and P.J. Kellogg, Electrostatic noise at the frequency beyond the Earth's bow shock, J. Geophys. Res., 84, 1369, 1979.

Fitzenreiter, R.J., A.J. Klimas, and J.D. Scudder, Detection of bump-on-tail reduced electron velocity distributions at the electron foreshock boundary, J. Geophys. Res., 11, 496, 1984.

Fitzenreiter, R.J., J.D. Scudder, and A.J. Klimas, Threedimensional analytical model for the spatial variation of the foreshock electron distribution function: Systematics and comparison with ISEE observations, $J$. Geophys. Res., 95, 4155, 1990.

Fuselier, S.A., D.A. Gurnett, and R.J. Fitzenreiter, The downshift of electron plasm oscillations in the electron foreshock region, J. Geophys. Res., 90, 3935, 1985.

Galeev, A.A., and R.Z. Sagdeev, Theory of weakly turbulent plasma, in Handbook of Plasma Physics, vol.1, edited by M. N. Rosenbluth, and R. Z. Sagdeev, p. 692, NorthHolland, New York, 1983.

Gary, P., Electrostatic instabilities in plasma with two electron components, J. Geophys. Res., 90, 9213, 1985.

Lacombe, C.A., C.C. Mangeney, C.C. Harvey, and J.D. Scudder, Electron plasma waves upstream of the Earth's bow shock, J. Geophys. Res., 90, 73, 1985.

Matsiborko N.G., Onischenko I.N., V.D. Shapiro, and V.I. Shevchenko, Nonlinear interaction of the monochromatic electron beam with the plasma particle, Plasma Phys., $14,597,1972$.

Marsh, E., Beam driven electron acoustic waves upstream of the Earth's bow shock, J. Geophys. Res., 90, 6327, 1985.

Mikhailovskii, A.B., Theory of Plasma Instabilities, vol.1, p.62, Consultants Bureau, New York, 1974.

Onsager, T.G. and R.H. Holzworth, Measurements of the electron beam mode in Earth's foreshock, J. Geophys. Res., 95, 4175, 1990.

Schriver, D. and M. Ashour-Abdalla, Broadband electrostatic noise due to field-aligned currents, J. Geophys. Res., $16,899,1989$.

P.M.E. Décréau, Laboratoire de Physique et chimie de l'Environnement terrestre et planétaire, 45071 Orléans Cédex 2, France. 
V. Fiala, Institute of Atmospheric Physics, Czech Academy of Physics, 14131 Prague 4, Czech Republic.

V. V. Pivovarov, Space Research Institute, 117810 Moscow GSP 17, Russia.

I. V. Shapiro, International Institute for Mathematical Geophysics, 113556 Moscow, Varshavskoe Shosse 79A, Russia.
V. I. Sotnikov, Institute of Geophysics and Planetary Physics, University of California, Los Angeles, CA 900241567.

(Received July 15, 1993; revised April 14, 1994; accepted May 18, 1994.) 